\title{
Macro-microscopic one-dimensional compression of wet granular soils by experimental investigation
}

\author{
Vinh-Du Than ${ }^{1,2, a}$, Patrick Aimedieu ${ }^{1}$, Jean-Michel Pereira ${ }^{1}$, Jean-Noël Roux ${ }^{1}$, Michel Bornert $^{1}$ and Anh-Minh Tang ${ }^{1}$ \\ ${ }^{1}$ Université Paris-Est, Laboratoire Navier (UMR 8205), CNRS, ENPC, IFSTTAR, 77455 Marne-la-Vallée Cedex 2, France \\ ${ }^{2}$ The University of Danang, College of Technology, Department of Civil Engineering, Danang City, Vietnam
}

\begin{abstract}
The presence of liquid menisci between soil particles in wet granular soils plays a key role in the macroscopic behavior. This liquid bridge between particles may also allow the existence of metastable microstructures that lead to mechanical instabilities when the soil in subjected to external loadings. In this work, the authors present an investigation into macroscopic and microscopic behaviors of wet granular soils in very loose state under one-dimensional (1D) compression by using oedometer test combined with the X-ray computed tomography (X-ray CT) observations. The specimen is prepared in the presence of a small amount of a water meniscus by using the controlled pluviation method. The influences of various mechanical parameters are first studied on the compression curve. The microstructure along those compression curves are then analyzed by using X-ray CT to observe the arrangement of microstructure under growing of applied force.
\end{abstract}

\section{Introduction}

Wet granular soils are usually found in many natural processes. In the field of geotechnical engineering, the appearance of water menisci between soil particles is the most important factor which can be governed the macroscopic behavior. Hence, the cohesive soils can be stable at very loose state however they are very sensitive to the external force. So far, there are a lot of studies in the macro-micromechanical behavior of wet granular materials, such as: cohesive soils [1,2], wet beads [3], loessic soils [4,5], wet sand [6,7], etc. In general, the behavior of this material under the growing of external load (oedometric or isotropic compression tests) is normally characterized by the irreversible compression and decompression curves.

Various investigations of the microstructure of loose unsaturated granular soils have been carried out by the scanning electron microscopy (SEM) $[3,4]$ and the microfocus X-ray computed tomography $(\mu \mathrm{CT})$ [7]. The SEM techniques require a cautious preliminary dehydration of the samples, often by freeze-drying. They provide local observations on a small part of millimetric samples. The $\mu \mathrm{CT}$ is a high-resolution non-destructive 3D observation technique that does not need any sample pre-treatment and that gives further $3 \mathrm{D}$ information on the whole sample.

Yet, wet granular soils, especially in very loose unsaturated states, have less frequently been investigated by the $\mu \mathrm{CT}$ than dense states. In this work, glass beads with capillary bonds in the pendular state were investigated by the oedometric compression tests and

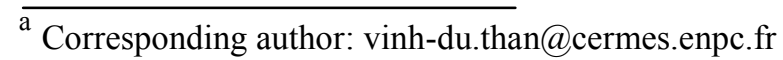

$\mu \mathrm{CT}$ tests for further macro-microscopic investigations of a wet bead sample.

\section{Material and methods}

\subsection{Material}

The studied material is glass beads, an industrial product, with the spherical beads. The size of diameters from 80 $\mu \mathrm{m}$ to $104 \mu \mathrm{m}$ is chosen for this study. With these diameters, particle weight looses relevance with respect to capillary forces [8], and they are large enough for the $\mathrm{X}$-ray CT tests. The specific density of this material was equal to $2,460 \mathrm{~kg} / \mathrm{m}^{3}$. The grain size distribution is shown in Figure 1.

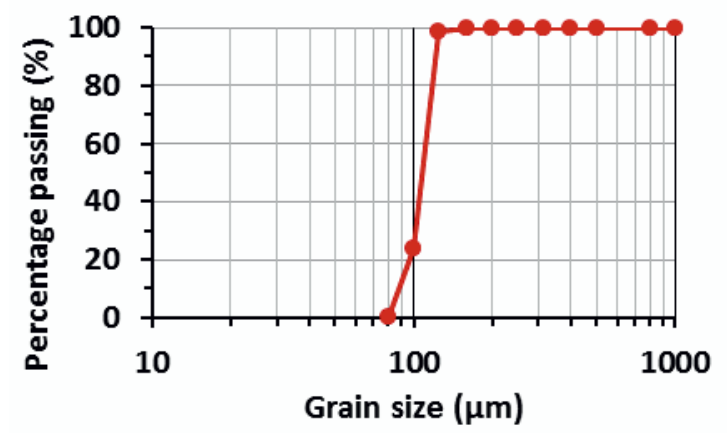

Figure 1. Cumulative grain size distribution of glass beads. 


\subsection{Specimen preparation}

The specimen was prepared as following steps:

- The glass beads were mixed with water at initial selected water content of $w_{0}=3.50 \%$, in order to ensure that the specimens were always maintained in pendular state.

- The oedometric cell $(50 \mathrm{~mm}$ of internal diameter for oedometer tests -- hereafter called cell $\phi 50$ and $20 \mathrm{~mm}$ of internal diameter for X-ray CT tests -hereafter called cell $\$ 20)$ was placed on the support system (see Figure 2). It was then filled with the wetted glass beads by using a controlled pluviation method as follows. The filling was performed by placing the wetted glass beads on a fixed sieve with a $200 \mu \mathrm{m}$ opening. This size allows passing for maximum of two grains. The sieve was vibrated vertically with a vibration machine. Thanks to the shocks, clusters of glass beads passed sequentially through the sieve to finally fall into the cell. The height of free fall $\left(h_{\text {fall }}\right)$ from bottom sieve to bottom of the cell was between 80 and $260 \mathrm{~mm}$.

- All specimens were prepared in an air-conditioned room. The preparation of each specimen took approximately 30 minutes. These conditions allow minimizing the water evaporation during the preparation.

- The specimen top surface was then perfected by using a small iron wire to cut the excess soil. This action must to do carefully and gently in order to avoid any perturbation to initial structure. Height of specimen is $20 \mathrm{~mm}$ (cell $\phi 50)$ and $10 \mathrm{~mm}$ (cellф20).

- Finally, all specimens were tested to measure the water content after preparation ( $w_{\text {ap }}$ ).

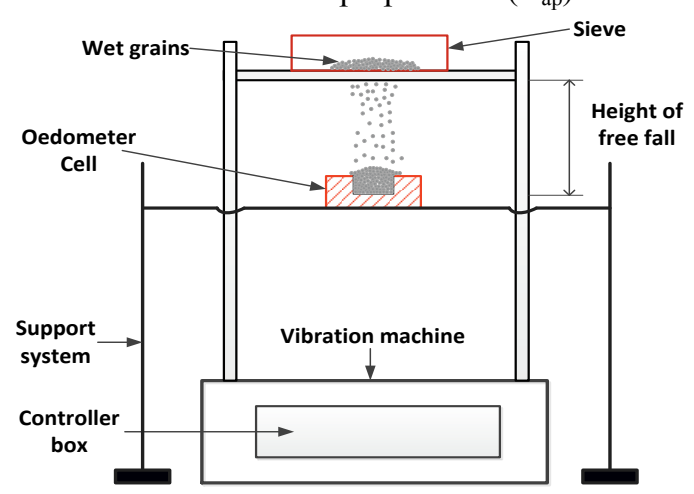

Figure 2. Schematic of system for preparing the specimen.

The initial parameters of samples after specimen preparation with cell $\$ 50$ and cell $\phi 20$, listed in Table 1.

The water content of all specimens after preparation is strongly affected by the height of free fall. Indeed, within same initial conditions, the evaporation of samples is proportional to the height of free fall. Clear evidences are given in Table 1, the maximal evaporation is $54.29 \%$ for highest height of $260 \mathrm{~mm}$ and minimal ones is 22.57 $\%$ corresponds to smallest height of $80 \mathrm{~mm}$. Although the water content after preparation is remained so small, these specimens are still stable at very loose state. This confirms that the capillary forces between grains play a key role to maintain the stability of specimen's structure. Moreover, the relationship between initial void ratio $e_{0}$ and the height of free fall $h_{\text {fall }}$ is shown in Figure 3, in which the blue square point is the sample for cell $\$ 20$. Indeed, the higher the heights of free fall, the smaller the initial void ratios.

Table 1. Initial parameters (height of free fall $h_{\text {fall }}$, porosity $n$, water content after preparation $w_{\text {ap }}$ and initial void ratio $e_{0}$ )

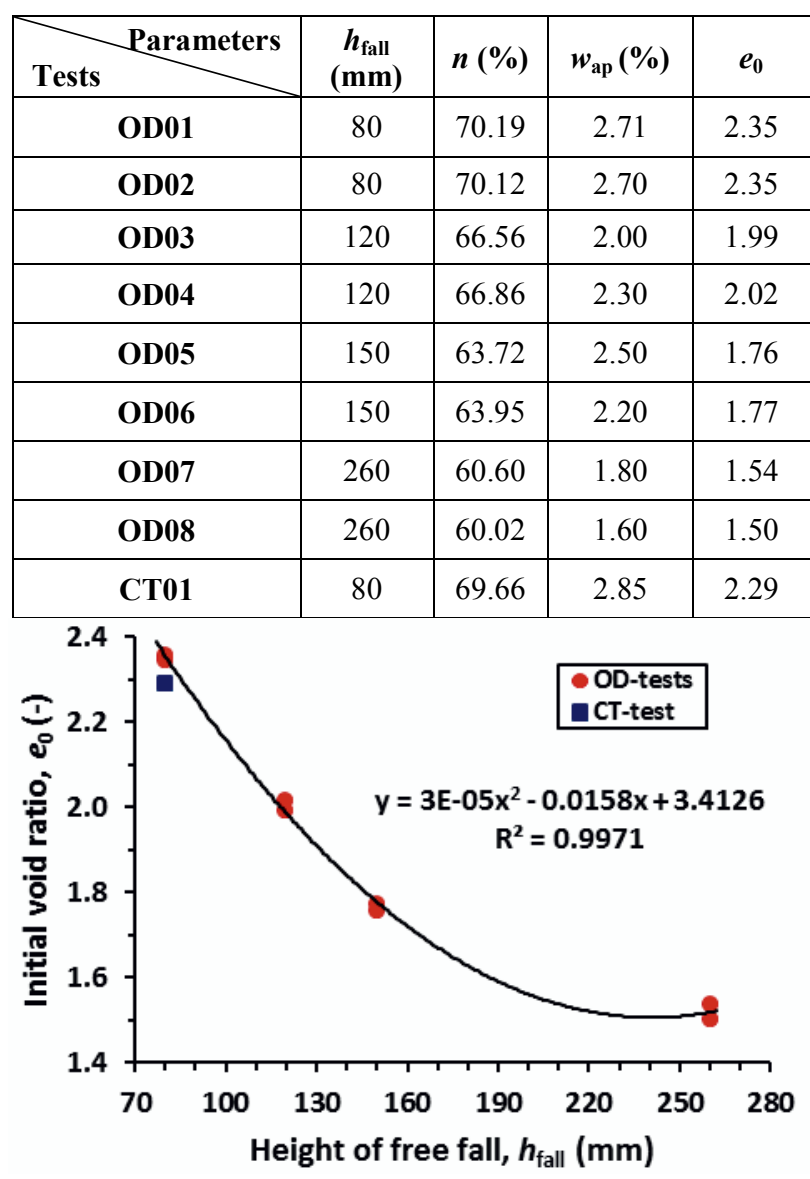

Figure 3. Initial void ratio $e_{0}$ versus height of free fall $h_{\text {fall }}$.

\subsection{Oedometric compression process}

The specimen was carefully placed on the loading platform of the compression system (see Figure 3). The piston is fixed to the load cell which has maximum capacity of 1,000 N. A LVDT (Linear Variable Differential Transformer) was installed to measure the displacement of specimen. The cell was then moved upward with a rate of $0.5 \mathrm{~mm} / \mathrm{min}$. All signals of load cell and LVDT were received by the data acquisition system using the LabVIEW software. 


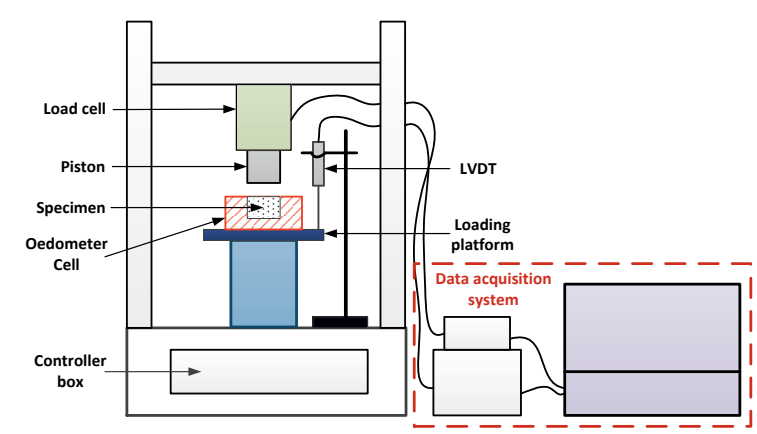

Figure 4. Schematic of oedometric compression system.

Figure 5 illustrates the typical relationship between applied forces and time during the compression process. Based on this curve, the initial vertical stress was specified after removing the noise's effect, especially the moment when the surface of specimen touched the piston. The settlement of specimens was then determined from the displacement of LVDT. The void ratio was finally calculated from the settlement and initial void ratio of specimen. All compacted specimens were also retested in order to measure the final height. This parameter was used to verify the final state of compression curve.

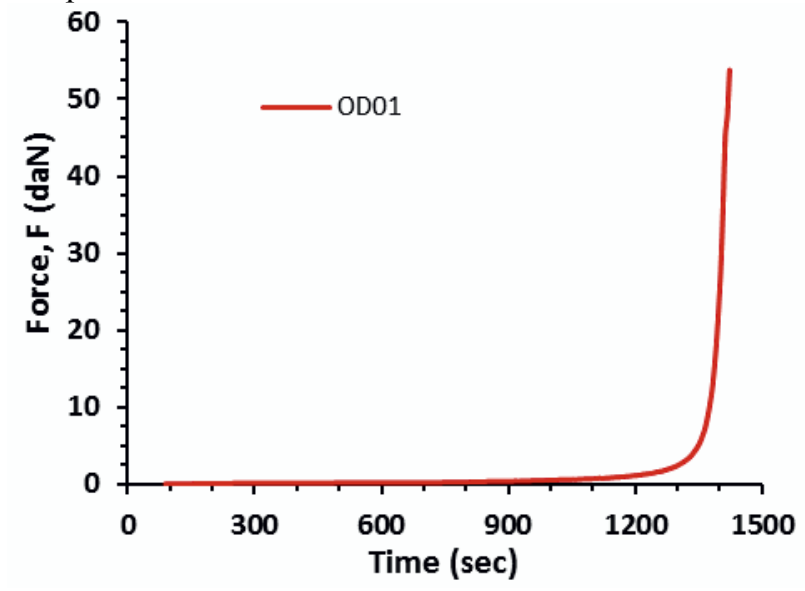

Figure 5. Applied force versus time during compaction process.

\subsection{X-ray computed tomography}

$\mathrm{X}$-ray computed tomography (X-ray CT) is a nondestructive method that allows to obtain threedimensional images of various materials (for more information on principles and applications, see $[9,10])$. It generally works well for porous media.

X-ray CT scans were performed using an UltraTom microtomograph, equipped with a $230 \mathrm{kV}$ micro-focus Hamamatsu L10801 source, associated with a PaxScan Varian $2520 \mathrm{~V}$ bidimentional sensor.

The specimen used for those scans was prepared in Sec. 2.2 with cell 20 . The cell was made of PMMA (PolyMethyl MethAcrylate).

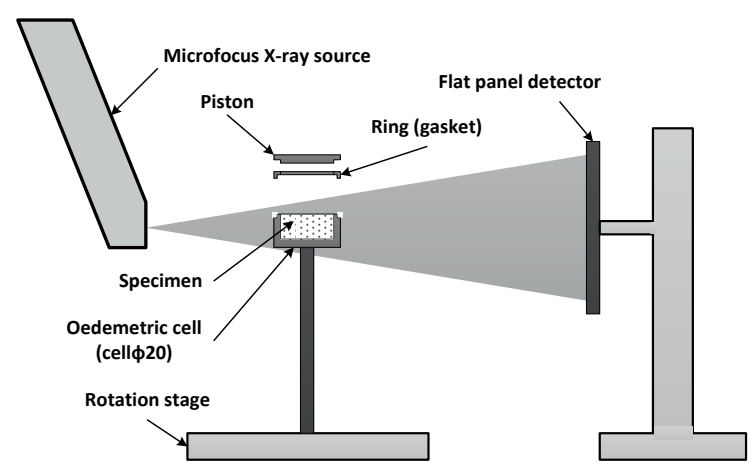

Figure 6. Schematic of the X-ray CT scanner.

Four scans (denoted by S1, S2, S3 and S4) were carried out, corresponding to four points on the compression curve. For each point, the position of the piston was fixed during scantime. Those four states represented different heights of the specimen: $h_{1}=10$ $\mathrm{mm}, h_{2}=8.7 \mathrm{~mm}, h_{3}=7.5 \mathrm{~mm}$ and $h_{4}=6.4 \mathrm{~mm}$. The corresponding the macroscopic void ratios were respectively calculated $e_{1}=2.29, e_{2}=1.87, e_{3}=1.47$ and $e_{4}=1.11$. X-ray source voltage and current were $70 \mathrm{kV}$ and $130 \mu \mathrm{A}$. Each scantime was $80 \mathrm{~min}$ long. Voxel size was $13 \mu \mathrm{m}$. In the present study, this resolution was applied just to analyze the homogeneity of specimen. Images were reconstructed using the Xact software (made by RX-Solutions). 3D-images are 16 bit-depth, and were converted to 8 bit-depth to reduce memory usage. The image analysis was carried out by using ImageJ [13] and FiJi softwares, which is another version ImageJ for 3D modeling [14].

\section{Results}

\subsection{Macroscopic behavior}

All the specimens were carried out with very loose initial structures, from $e_{0}=2.35$ to $e_{0}=1.50$. Figure 7 shows the compression curves for different values of initial void ratios. All the tests were duplicated to ensure the repetition. The initial stress at bottom with the weight of sample is $0.246 \mathrm{kPa}$.

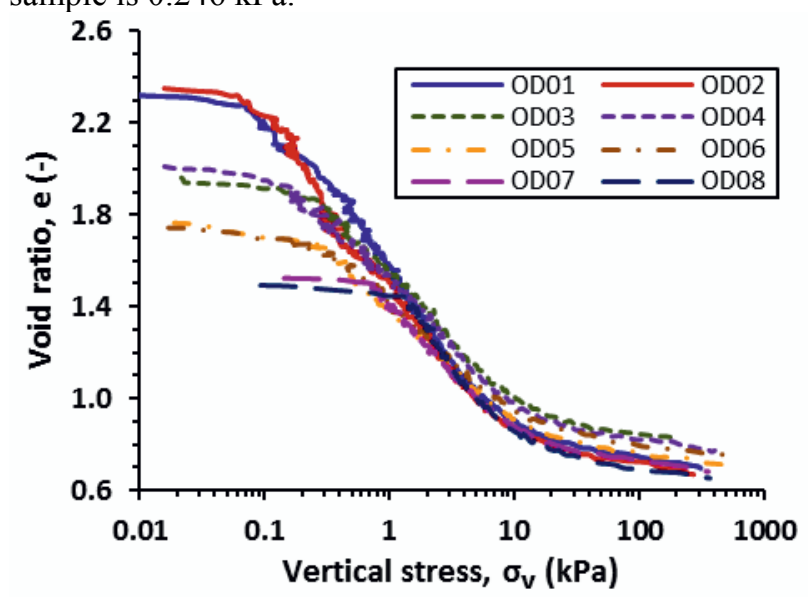

Figure 7. Compression curves for different values of initial void ratios. 
Based on the shape of these curves and the range of stress, three stages can be identified. The first stage, under the low stress, void ratio $e$ was slightly decreased. The void ratio was then sharply decreased in the intermediate stage. Finally, at third stage, void ratio was gradually decreased when the vertical stress was larger than $10 \mathrm{kPa}$. Especially, these curves were very close in this stage. In other words, when no further rearrangement is possible, the decrease of void ratio was very small because of the elastic deformation of grains at the contact points. Interestingly, there were only two stages 1 and 2 on the oedometric compression curve in the usual soil mechanics behavior $[4,11]$. This is a special behavior of the studied material under the 1D compression tests.

\subsection{Microscopic behavior}

The reconstructed 3D sample volume at initial state is shown in Figure 8. $\mu$ CT technique allows distinguishing between various components, according to their density and atomic composition, thanks to grey levels. On examining reconstructed pictures, lighter grey levels typically correspond to more absorbing materials, while darker grey levels corresponds to less absorbing materials.

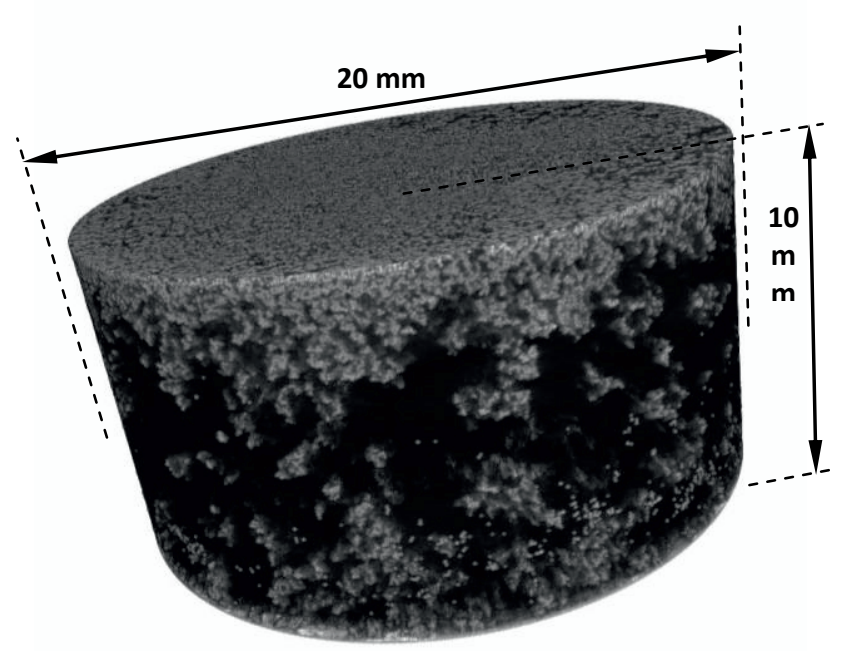

Figure 8. 3D reconstructed image of the sample at initial void ratio $(\mathrm{S} 1)$.

The density is not homogeneous within the cylindrical sample, especially at the periphery where more voids are observed plus a larger density with less voids at top and bottom of sample. This is due to the specimen preparation, and also to the boundary conditions imposed by the ring. In other words, the friction between the ring and grains and the cohesion of grains resisting displacement the other particles.

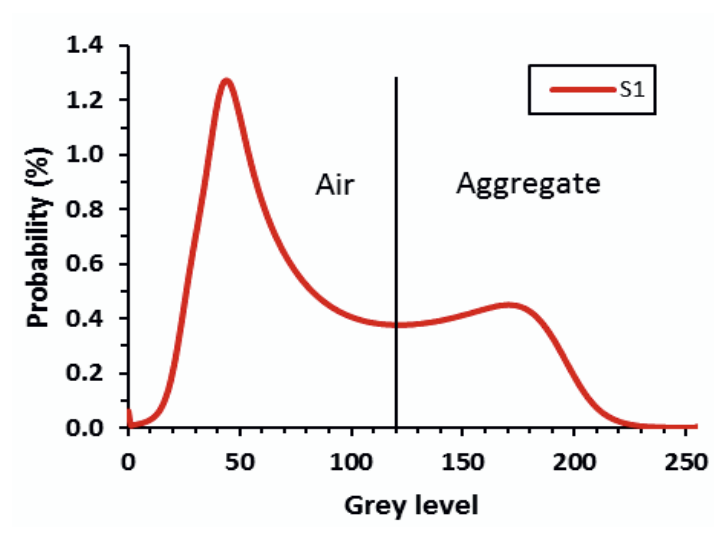

Figure 9. Grey level histogram for the scan S1.

Figure 9 illustrates the representative histograms of grey level for the first scans. Grey levels range from 0 to 255. A threshold, shown on the same figure, was used to delineate o border between air dominated portions of the sample (left of the line) and aggregates. The grey-level threshold value was found in the interval [110:130]. This allowed recalculating the total void ratio, as well as the void ratio distribution during the compaction process. The IsoData method [12] was chosen for the segmentation process. The grey-level threshold values following 128, 122, 130 and 128 were selected for scans $\mathrm{S} 1, \mathrm{~S} 2, \mathrm{~S} 3$ and S4, respectively.

Figure 10 shows the segmentation process corresponding to three consecutive stages: a) initial state; b) during the thresholding process and c) segmented image. After thresholding, the aggregates (darker grey levels) and the air (lighter grey levels) were clearly distinguished. The void ratio was then recalculated by dividing the number of black voxels by the total number of voxels of the segmented image.

(a)

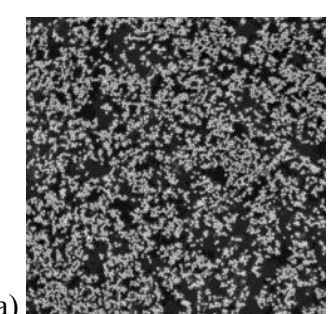

(b)
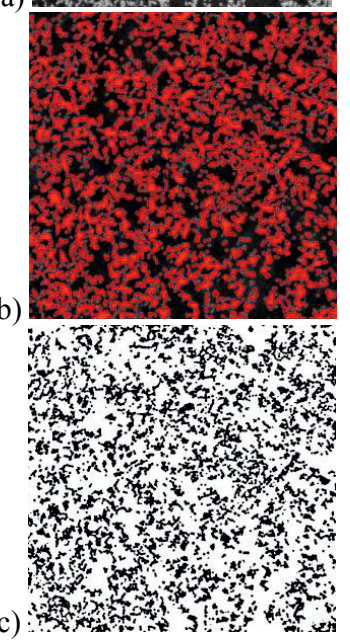

Figure 10. Images at different steps of segmentation: (a) original image, (b) during the thresholding process and (c) segmented image (binary image). 
The void ratio distribution according to the height of the specimen during the compression process is illustrated in Figure 11. Ten layers of sample are split corresponding to the same thickness from first layer $\mathrm{h}_{1}$ to last layer $\mathrm{h}_{10}$ (from bottom to top), as described in the inset figure.

At the initial state $\mathrm{S} 1$, from bottom to top, the void ratio is largest at sixth layer $\left(e_{\max }=2.35\right)$ because of the influence of the wall in specimen preparation process. Meanwhile the sample is denser at the bottom layers and densest at the top layers. This is caused by the end of preparation process when the grains were spread on the surface of the cell, no longer the wall's effect. The lower layers were steadily compacted by the grains above them. This confirms that the void ratio at this region is smallest. For the step 2 (S2), the structure is compacted and rearranged, void ratio in the central and top layers is reduced, especially the central layers. The structure is notably perturbed in step 3 (S3). The void ratio is reduced of $49.80 \%$ compared to its at bottom layers and of 23.25 $\%$ at the top layers. At the densest state (S4), a strong decrease of void ratio obtained at the central layers, while this void ratio is very close to it's at the bottom layer $\left(\mathrm{h}_{1}\right)$. The average void ratio of four curves $(2.31,1.91,1.48$ and 1.14 , corresponding to S1, S2, S3 and S4) are very close to the macroscopic ones, which are given in Sec. 2.4 .

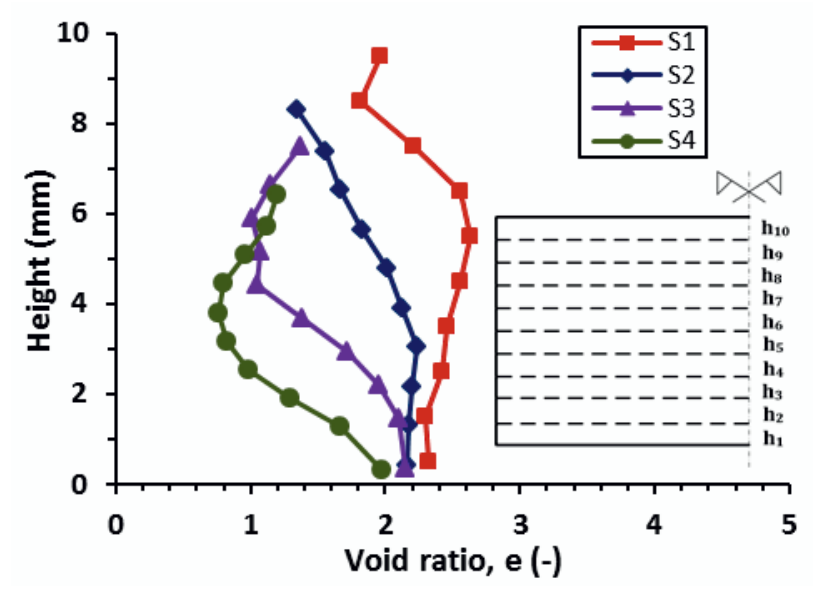

Figure 11. Void ratio distribution according to the height of sample for different compaction steps.

A supplemental evidence for the void ratio distribution during the compaction process is shown in Figure 12.

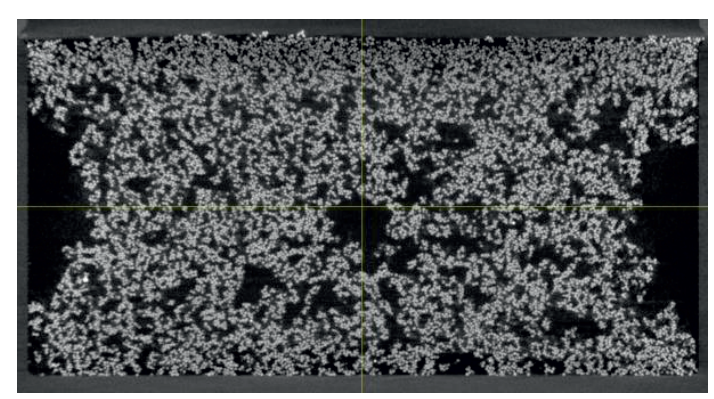

(S1)

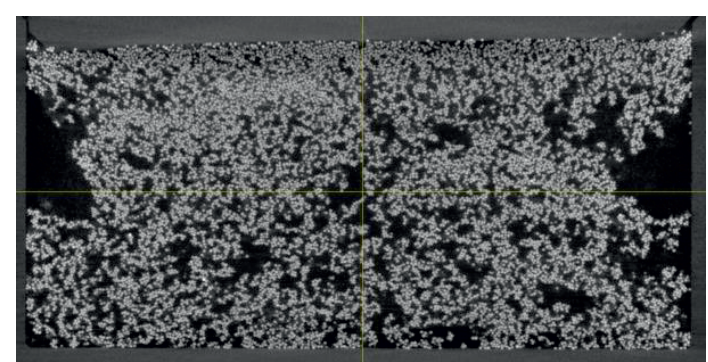

(S2)

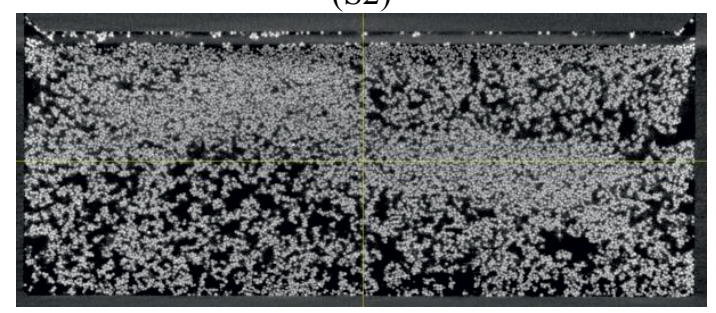

(S3)

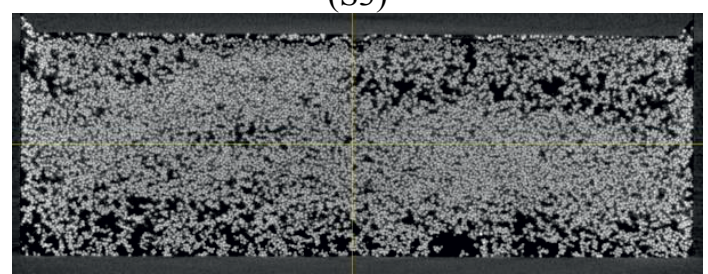

(S4)

Figure 12. Vertical slice at the center of the sample for 4 states.

Figure 12 clearly shows the void ratio distribution during the compaction process along the vertical direction. Under a growing stress, the structure is strongly rearranged in the central layers because the loosest state is obtained in this region while it is slightly affected at the bottom and top layers. When the specimen becomes denser in central, it can be against the settlement of top layers. Moreover, relying on the cohesive forces, the grains at bottom layers can be create arches which strongly support above structures. This explains why it is really difficult to compact this specimen at step 4 although volumetric strain is only $40 \%$.

In addition, the spatial distribution of the void ratio is investigated as a radial function, considering different concentric cylinders having the same height for each scan. Ten concentric cylinders from the center (smallest radius $R_{1}=0.5 \mathrm{~mm}$ ) to the contour (radius $R_{10}$ of $9.5 \mathrm{~mm}$, see Figure 13-a).

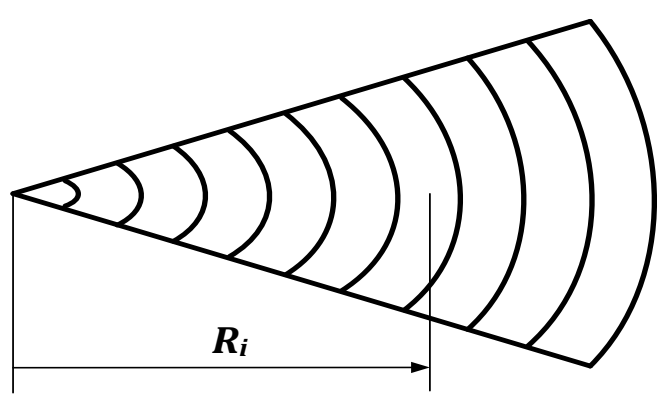

(a) 


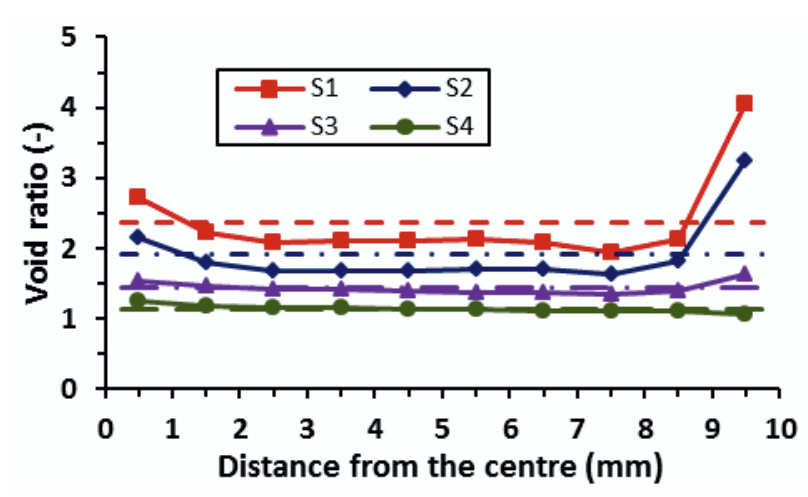

(b)

Figure 13. (a) Concentric cylinders investigated. (b) The change of void ratio respects to the average radii of the selected cylinders.

The calculated void ratios correspond to the cylinders for 4 steps of compaction process, as shown in Figure 13b. Each curve, illustrates the radial void variation for each of the four steps. The mean distribution of void is rather regular, except for the first and the last point of step 1 and step 2. Void ratios are larger at the center and periphery of the cylinders, especially in steps 1 and 2 . Once again, this demonstrates the remarkable effect of the wall which is already mentioned above. Furthermore, during the specimen preparation, the grains firstly fell into the center of the cell. They were then dispersed around until spread to the cell. This leads to the existence of many macro pores in cylinder $\mathrm{R}_{1}$. Hence, under low stress, the void ratio in this cylinder is larger than the void ratios in the intermediate cylinders (see the first point of two curves S1 and S2).

In spite of the precautions and carefulness taken during the specimen preparation, $\mu \mathrm{CT}$ clearly demonstrated that the sample was heterogeneous with looser zones in the center and the periphery. More complete analyses of the corresponding microstructural changes are being carried out.

\section{Conclusions and Perspectives}

These results show that a very loose yet stable-state of wet granular soils can be made for tests, even using very small water content. Besides, the oedometric tests exhibit three stages of compression curve of cohesive soils with different values of low initial densities. This is not existent in the usual soil mechanics.

$\mu \mathrm{CT}$ investigations of the microstructure allowed quantifying the degree of homogeneity and the arching effect at the bottom of sample by combining with the image processing.

The research program developed here can be extended into several directions. For example, the pore size distribution during the compaction test, images will be analyzed by using the Volumetric Digital Image Correlation tools. A comparison with simulation results will be mentioned for macro-microscopic behaviors.

\section{Acknowledgments}

The first author of the paper is supported by the Scholarship (the Project No 911) of Ministry of Education and Training, the Vietnamese Government to do this work.

\section{References}

[1] D. M. Wood, Soil Behaviour and Critical State Soil Mechanics (Cambridge University Press, 1990).

[2] J. K. Mitchell and K. Soga, Fundamentals of Soil Behavior (Wiley, 1976).

[3] Z. Fournier, D. Geromichalos, S. Herminghaus, M. M. Kohonen, F. Mugele, M. Scheel, M. Schulz, B. Schulz, R. Seemann, and A. Skudelny, J. Phys. Condens. Matter 17, 477 (2005).

[4] J. Munõz-Castelblanco, P. Delage, J.-M. Pereira, and Y. J. Cui, Géotechnique Lett. 1, 17 (2011).

[5] M. Jiang, H. Hu, and F. Liu, Can. Geotech. J. 1157, 1147 (2012).

[6] N. Mitarai and F. Nori, Adv. Phys. 00, 1 (2006).

[7] J.-F. Bruchon, J.-M. Pereira, M. Vandamme, N. Lenoir, P. Delage, and M. Bornert, Granul. Matter 15, 783 (2013).

[8] J. C. Santamarina, in Soil Behav. Soft Gr. Constr., edited by C. C. Ladd (2001), pp. 1-32.

[9] J. Banhart, Advanced Tomographic Methods in Materials Research and Engineering (Oxford University Press, New York, 2008).

[10] J. Hsied, Computed Tomography - Principles, Design, Artifacts, and Recent Advances, Second Edi (SPIE and John Wiley \& Sons, Inc., United States of America, 2009).

[11] A. M. Tang, Y. J. Cui, J. Eslami, and P. Défossez, Geoderma 148, 282 (2009).

[12] S. Ridler, T.W. Calvard, IEEE Trans. Syst. Man Cybern. 8, 630 (1978).

[13] http://imagej.nih.gov/ij/index.html

[14] http://fiji.sc/Fiji 